\title{
Exponential stability criteria for fuzzy bidirectional associative memory Cohen-Grossberg neural networks with mixed delays and impulses
}

Weina He and Longxian Chu*

\section{"Correspondence:}

chulongxian_pdsu@126.com Software College, Pingdingshan University, Pingdingshan, 467000 PR China

\section{然 Springer}

\begin{abstract}
This paper is concerned with fuzzy bidirectional associative memory (BAM) Cohen-Grossberg neural networks with mixed delays and impulses. By constructing an appropriate Lyapunov function and a new differential inequality, we obtain some sufficient conditions which ensure the existence and global exponential stability of a periodic solution of the model. The results in this paper extend and complement the previous publications. An example is given to illustrate the effectiveness of our obtained results.
\end{abstract}

MSC: 34C20; 34K13; $92 \mathrm{~B} 20$

Keywords: fuzzy BAM Cohen-Grossberg neural networks; exponential stability; mixed delays; periodic solution; impulse

\section{Introduction}

In recent years, considerable attention has been paid to bidirectional associative memory (BAM) Cohen-Grossberg neural networks [1] due to their potential applications in various fields such as neural biology, pattern recognition, classification of patterns, parallel computation and so on [2-4]. In real life, numerous application examples appear, for example, emerging parallel/distributed architectures were explored for the digital VLSI implementation of adaptive bidirectional associative memory (BAM) [5], Teddy and Ng [6] applied a novel local learning model of the pseudo self-evolving cerebellar model articulation controller (PSECMAC) associative memory network to produce accurate forecasts of ATM cash demands. Chang et al. [7] proposed a maximum-likelihood-criterion based on BAM networks to evaluate the similarity between a template and a matching region. Sudo et al. [8] proposed a novel associative memory that operated in noisy environments and performed well in online incremental learning applying self-organizing incremental neural networks. On the one hand, the existence and stability of the equilibrium point of BAM Cohen-Grossberg neural networks plays an important role in practical application. On the other hand, time delay is inevitable due to the finite switching speed of amplifiers in the electronic implementation of analog neural networks, moreover, time delays may have important effect on the stability of neural networks and lead to periodic oscillation,

(c) The Author(s) 2017. This article is distributed under the terms of the Creative Commons Attribution 4.0 International License (http://creativecommons.org/licenses/by/4.0/), which permits unrestricted use, distribution, and reproduction in any medium, provided you give appropriate credit to the original author(s) and the source, provide a link to the Creative Commons license, and indicate if changes were made. 
bifurcation, chaos and so on $[3,9,10]$. Thus many interesting stability results on BAM Cohen-Grossberg neural networks with delays have been available [11-30].

As is well known, numerous dynamical systems of electronic networks, biological neural networks, and engineering fields often undergo abrupt change at certain moments due to instantaneous perturbations which leads to impulsive effects $[4,19,20,31-36]$. Many scholars $[37,38]$ think that uncertainty or vagueness often appear in mathematical modeling of real world problems, thus it is necessary to take vagueness into consideration. Fuzzy neural networks (FNNs) pay an important role in image processing and pattern recognition [37] and some results have been reported on stability and periodicity of FNNs [11, 3941]. Here we would like to point out that most neural networks involve negative feedback terms and do not possess amplification functions or behaved functions. The model (1.1) of this paper has amplifications function and behaved functions which differ from most neural networks with negative feedback term. Up to now, there are rare papers that consider exponential stability of this kind of fuzzy bidirectional associative memory CohenGrossberg neural networks with mixed delays and impulses.

Inspired by the discussion above, in this paper, we are to consider the following fuzzy bidirectional associative memory Cohen-Grossberg neural networks with mixed delays and impulses,

$$
\left\{\begin{aligned}
\dot{x}_{i}(t)= & l_{i}\left(x_{i}(t)\right)\left[-a_{i}\left(t, x_{i}(t)\right)+\sum_{j=1}^{m} c_{j i}(t) f_{j}\left(y_{j}(t-\tau(t))\right)\right. \\
& +\bigwedge_{j=1}^{m} \alpha_{j i}(t) \int_{-\infty}^{t} K_{j i}(t-s) f_{j}\left(y_{j}(s)\right) d s+\bigwedge_{j=1}^{m} T_{j i} u_{j}+\bigvee_{j=1}^{m} H_{j i} u_{j} \\
& \left.+\bigvee_{j=1}^{m} \beta_{j i}(t) \int_{-\infty}^{t} K_{j i}(t-s) f_{j}\left(y_{j}(s)\right) d s+I_{i}(t)\right], \quad t \neq t_{k}, i \in \ell, \\
\triangle x_{i}\left(t_{k}\right) & =x_{i}\left(t_{k}\right)-x_{i}\left(t_{k}^{-}\right)=-\gamma_{i k} x_{i}\left(t_{k}^{-}\right)+\sum_{j=1}^{m} e_{i j}\left(t_{k}^{-}\right) E_{j}\left(y_{j}\left(t_{k}^{-}-\tau\right)\right), \quad k \in \mathbb{Z}_{+}, \\
\dot{y}_{j}(t)= & \vartheta_{j}\left(y_{j}(t)\right)\left[-b_{j}\left(t, y_{j}(t)\right)+\sum_{i=1}^{n} d_{i j}(t) g_{i}\left(x_{i}(t-\tau(t))\right)\right. \\
& +\bigwedge_{i=1}^{n} p_{i j}(t) \int_{-\infty}^{t} N_{i j}(t-s) g_{i}\left(x_{i}(s)\right) d s+\bigwedge_{i=1}^{n} S_{i j} u_{i}+\bigvee_{i=1}^{n} L_{i j} u_{i} \\
& \left.+\bigvee_{i=1}^{n} q_{i j}(t) \int_{-\infty}^{t} N_{i j}(t-s) g_{i}\left(x_{i}(s)\right) d s+J_{j}(t)\right], \quad t \neq t_{k}, j \in \hbar, \\
\triangle y_{j}\left(t_{k}\right) & =y_{j}\left(t_{k}\right)-y_{j}\left(t_{k}^{-}\right)=-\delta_{j k} y_{i}\left(t_{k}^{-}\right)+\sum_{i=1}^{n} h_{j i}\left(t_{k}^{-}\right) H_{i}\left(x_{i}\left(t_{k}^{-}-\tau\right)\right), \quad k \in \mathbb{Z}_{+},
\end{aligned}\right.
$$

with initial conditions

$$
\begin{cases}x_{i}(s)=\phi_{1 i}(s), & s \in(-\infty, 0], i \in \ell, \\ y_{j}(s)=\phi_{2 i}(s), & s \in(-\infty, 0], j \in \hbar,\end{cases}
$$

where $n$ and $m$ correspond to the number of neurons in $X$-layer and $Y$-layer, respectively. $x_{i}(t)$ and $y_{j}(t)$ are the activations of the $i$ th neuron and the $j$ th neurons, respectively. $\iota_{i}(\cdot)$ and $\vartheta_{j}(\cdot)$ are the abstract amplification functions, $a_{i}(t, \cdot)$ and $b_{j}(t, \cdot)$ stand for the rate functions with which the $i$ th neuron and $j$ th neuron will reset its potential to the resting state in isolation when disconnected from the network and external inputs; $\alpha_{j i}(t), \beta_{j i}(t), T_{j i}$ and $H_{j i}$ are elements of fuzzy feedback MIN template and fuzzy feedback MAX template, fuzzy feed-forward MIN template and fuzzy feed-forward MAX template in $X$-layer, respectively; $p_{i j}(t), q_{i j}(t), S_{i j}$ and $L_{i j}$ are elements of fuzzy feedback MIN template and fuzzy feedback MAX template, fuzzy feed-forward MIN template and fuzzy feed-forward MAX template in $Y$-layer, respectively; $\bigwedge$ and $\bigvee$ denote the fuzzy AND and fuzzy OR operation, respectively; $u_{j}, u_{i}$ denote external input of the $i$ th neurons in $X$-layer and external input of the $j$ th neurons in $Y$-layer, respectively; $I_{i}(t)$ and $J_{j}(t)$ are external bias of $X$-layer and $Y$ layer, respectively, $f_{j}(\cdot)$ and $g_{i}(\cdot)$ are signal transmission functions, $K_{j i}(t)$ and $N_{i j}(t)$ are delay 
kernels, $\ell=\{1,2, \ldots, n\}, \hbar=\{1,2, \ldots, m\}, \mathbb{Z}_{+}$denotes the set of positive integral numbers, the impulse times $t_{k}$ satisfy $0=t_{0}<t_{1}<t_{2}<\cdots<t_{k}<\cdots, \lim _{k \rightarrow \infty} t_{k}=\infty, \phi_{1 i}(\cdot), \phi_{2 j}(\cdot) \in \mathbb{C}$, where $\mathbb{C}$ denotes real-valued continuous functions defined on $(-\infty, 0], \tau(t)$ is the transmission delay such that $0 \leq \tau(t) \leq \tau, \tau$ is a positive constant, $e_{i j}\left(t_{k}^{-}\right)$represents impulsive perturbations of the $i$ th unit at time $t_{k}, h_{j i}\left(t_{k}^{-}\right)$represents impulsive perturbations of the $j$ th unit at time $t_{k}, E_{j}\left(y_{j}\left(t_{k}^{-}\right)\right)$represents impulsive perturbations of the $j$ th unit at time $t_{k}$ and $y_{j}\left(t_{k}^{-}\right)$denotes impulsive perturbations of the $j$ th unit at time $t_{k}$ caused by the transmission delays, $H_{i}\left(x_{i}\left(t_{k}^{-}\right)\right)$represents impulsive perturbations of the $i$ th unit at time $t_{k}$ and $x_{i}\left(t_{k}^{-}\right)$denotes impulsive perturbations of the $i$ th unit at time $t_{k}$ which caused by the transmission delays. For details, see [42-44].

The main purpose of this paper is to investigate the existence and global exponential stability of a periodic solution of fuzzy BAM Cohen-Grossberg neural networks with mixed delays and impulses. By constructing a suitable Lyapunov function and a new differential inequality, we establish some sufficient conditions to ensure the existence and global exponential stability of a periodic solution of the model (1.1). The results obtained in this paper extend and complement the previous studies in $[4,10]$. Two examples are given to illustrate the effectiveness of our theoretical findings. To the best of our knowledge, there are very few papers that deal with this aspect. Therefore we think that the study of the fuzzy BAM Cohen-Grossberg neural networks with mixed delays and impulses has important theoretical and practical value. Here we shall mention that since the existence of amplifications function and behaved functions in model (1.1), thus there are some difficulties in dealing with the exponential stability. We will apply some inequality techniques, meanwhile, the construction of Lyapunov function is a key issue.

The remaining part of this paper is organized as follows. In Section 2, the necessary definitions and lemmas are introduced. In Section 3, we present some new sufficient conditions to ensure the existence and global exponential stability of a periodic solution of model (1.1). In Section 4, an illustrative example is given to show the effectiveness of the proposed method. A brief conclusion is drawn in Section 5 .

\section{Preliminaries}

Let $\mathbb{R}$ denote the set of real number, $\mathbb{R}^{n}$ the $n$-dimensional real space equipped with the Euclidean norm $|\cdot|, \mathbb{R}_{+}$the set of positive numbers. Denote $P C\left(\mathbb{R}, \mathbb{R}_{+}\right)=\left\{\phi: \mathbb{R} \rightarrow \mathbb{R}^{n}\right.$ : $\phi(t)$ is continuous for $t \neq t_{k}, \phi\left(t_{k}^{+}\right), \phi\left(t_{k}^{-}\right) \in \mathbb{R}^{n}$ and $\left.\phi\left(t_{k}^{-}\right)=\phi\left(t_{k}\right)\right\}$.

Throughout this paper, we make the following assumptions:

(H1) For $i \in \ell, j \in \hbar, c_{j i}(t), \alpha_{j i}(t), \beta_{j i}(t), e_{i j}(t), d_{i j}(t), p_{i j}(t), q_{i j}(t), h_{j i}(t), \tau(t), I_{i}(t)$ and $J_{j}(t)$ are all continuously periodic functions defined on $t \in[0, \infty)$ with common period $\omega>0$.

(H2) For $i \in \ell, j \in \hbar$, there exist positive constants $L_{j}^{f}, L_{j}^{E}, L_{i}^{g}$ and $L_{j}^{H}$ such that

$$
\begin{array}{lc}
\left|f_{j}(u)-f_{j}(v)\right| \leq L_{j}^{f}|u-v|, & \left|E_{j}(u)-E_{j}(v)\right| \leq L_{j}^{E}|u-v|, \\
\left|g_{i}(u)-g_{i}(v)\right| \leq L_{i}^{g}|u-v|, & \left|H_{i}(u)-H_{i}(v)\right| \leq L_{j}^{H}|u-v|
\end{array}
$$

for all $u, v \in \mathbb{R}$.

(H3) For $i \in \ell, j \in \hbar, \iota_{i}(\cdot)$ and $\vartheta_{j}(\cdot)$ are continuous and satisfy $0 \leq \underline{\iota}_{i} \leq \iota_{i}(\cdot) \leq \bar{\iota}_{i}$, $0 \leq \underline{\vartheta}_{j} \leq \vartheta_{j}(\cdot) \leq \bar{\vartheta}_{j}$, where $\underline{\iota}_{i}, \bar{l}_{i}, \underline{\vartheta}_{j}, \bar{\vartheta}_{j}$ are some positive constants. 
(H4) For $i \in \ell, j \in \hbar$, there exist continuous positive $\omega$-periodic functions $\varrho_{i}(t)$ and $\sigma_{j}(t)$ such that

$$
\frac{a_{i}(t, u)-a_{i}(t, v)}{u-v} \geq \varrho_{i}(t), \quad \frac{b_{j}(t, u)-b_{j}(t, v)}{u-v} \geq \sigma_{j}(t)
$$

for all $u, v \in \mathbb{R}$.

(H5) For $i \in \ell, j \in \hbar$, the delay kernels $K_{i j}(\cdot), N_{j i}(\cdot) \in C\left(\mathbb{R}^{+}, \mathbb{R}^{+}\right)$are piecewise continuous and satisfy $K_{i j}(s) \leq \tilde{K}(s)$ and $N_{j i}(s) \leq \tilde{K}(s)$ for all $s \in \mathbb{R}^{+}$, where $\tilde{K}(s) \in C\left(\mathbb{R}^{+}, \mathbb{R}^{+}\right)$ and integrable, satisfying $\int_{0}^{\infty} \tilde{K}(s) e^{\mu s} d s<\infty$, in which the constant $\mu$ denotes some positive number.

(H6) For $i \in \ell, j \in \hbar \omega>0$, there exists $q \in \mathbb{Z}^{+}$such that $t_{k}+\omega=t_{k+q}$ and $\gamma_{i k}=\gamma_{i(k+q)}$, $\delta_{j k}=\delta_{j(k+q)}, k \in \mathbb{Z}^{+}$.

(H7) For $i \in \ell, j \in \hbar, c_{j i}^{*}=\max _{t \in[0, \omega]}\left|c_{j i}(t)\right|, \alpha_{j i}^{*}=\max _{t \in[0, \omega]}\left|\alpha_{j i}(t)\right|, \beta_{j i}^{*}=\max _{t \in[0, \omega]}\left|\beta_{j i}(t)\right|$, $e_{i j}^{*}=\max _{t \in[0, \omega]}\left|e_{i j}(t)\right|, d_{i j}^{*}=\max _{t \in[0, \omega]}\left|d_{i j}(t)\right|, p_{i j}^{*}=\max _{t \in[0, \omega]}\left|p_{i j}(t)\right|$, $q_{i j}^{*}=\max _{t \in[0, \omega]}\left|q_{i j}(t)\right|, h_{j i}^{*}=\max _{t \in[0, \omega]}\left|h_{j i}(t)\right|, \varrho_{i}^{\star}=\min _{t \in[0, \omega]}\left|\varrho_{i}(t)\right|$, $\sigma_{i}^{\star}=\min _{t \in[0, \omega]}\left|\sigma_{j}(t)\right|$.

In this paper, we use the following norm of $\mathbb{R}^{n+m}$ :

$$
\|u\|=\sum_{i=1}^{n}\left|x_{i}\right|+\sum_{j=1}^{m}\left|y_{j}\right|, \quad\|\phi\|=\sup _{s \in(-\infty, 0]}\left[\sum_{i=1}^{n}\left|\phi_{1 i}(s)\right|+\sum_{j=1}^{m}\left|\phi_{2 j}(s)\right|\right]
$$

for $u=\left(x_{1}, x_{2}, \ldots, x_{n}, y_{1}, y_{2}, \ldots, y_{m}\right)^{T} \in \mathbb{R}^{n+m}, \phi=\left(\phi_{11}, \phi_{12}, \ldots, \phi_{1 n}, \phi_{21}, \phi_{22}, \ldots, \phi_{2 m}\right)^{T} \in \mathbb{C}^{n+m}$

Lemma 2.1 ([37]) Let $x$ and $y$ be two states of system (1.1). Then

$$
\left|\bigwedge_{j=1}^{n} \alpha_{i j}(t) g_{j}(x)-\bigwedge_{j=1}^{n} \alpha_{i j}(t) g_{j}(y)\right| \leq \sum_{j=1}^{n}\left|\alpha_{i j}(t)\right|\left|g_{j}(x)-g_{j}(y)\right|
$$

and

$$
\left|\bigvee_{j=1}^{n} \beta_{i j}(t) g_{j}(x)-\bigvee_{j=1}^{n} \beta_{i j}(t) g_{j}(y)\right| \leq \sum_{j=1}^{n}\left|\beta_{i j}(t)\right|\left|g_{j}(x)-g_{j}(y)\right| .
$$

Lemma $2.2([4])$ Let $p, q$, $r$ and $\tau$ denote nonnegative constants and $f \in P C\left(\mathbb{R}, \mathbb{R}_{+}\right)$satisfies the scalar impulsive differential inequality

$$
\left\{\begin{array}{l}
D^{+} f(t) \leq-p f(t)+q \sup _{t-\tau \leq s \leq t} f(s)+r \int_{0}^{\sigma} k(s) f(t-s) d s, \quad t \neq t_{k}, t \geq t_{0} \\
f\left(t_{k}\right) \leq a_{k} f\left(t_{k}^{-}\right)+b_{k} f\left(t_{k}^{-}-\tau\right), \quad k \in \mathbb{Z}^{+},
\end{array}\right.
$$

where $0<\sigma \leq+\infty, a_{k}, b_{k} \in \mathbb{R}, k(\cdot) \in P C\left([0, \sigma], \mathbb{R}^{+}\right)$satisfies $\int_{0}^{\sigma} k(s) e^{\eta_{0} s} d s<\infty$ for some positive constant $\eta_{0}>0$ in this case when $\sigma=+\infty$. Moreover, when $\sigma=+\infty$, the interval $[t-\sigma, t]$ is understood to be replaced by $(-\infty, t]$. Assume that (i) $p>q+r \int_{0}^{\sigma} k(s) d s$.

(ii) There exist constant $M>0, \eta>0$ such that

$$
\prod_{k=1}^{n} \max \left\{1, a_{k}+b_{k} e^{\lambda \tau}\right\} \leq M e^{\eta\left(t_{n}-t_{0}\right)}, \quad n \in \mathbb{Z}_{+},
$$


where $\lambda \in\left(0, \eta_{0}\right)$ satisfies

$$
\lambda<p-q e^{\lambda \tau}-r \int_{0}^{\sigma} k(s) e^{\lambda s} d s
$$

Then

$$
f(t) \leq M \bar{f}\left(t_{0}\right) e^{-(\lambda-\eta)\left(t-t_{0}\right)}, \quad t \geq t_{0}
$$

where $\bar{f}\left(t_{0}\right)=\sup _{t_{0}-\max \{\sigma, \tau\}} f(s)$.

\section{Global exponential stability of the periodic solution}

In this section, we will discuss the global exponential stability of the periodic solution for (1.1).

Theorem 3.1 Assume that (H1)-(H7) hold, then there exists a unique $\omega$-periodic solution of system (1.1) which is globally exponentially stable if the following conditions are fulfilled.

(H8)

$$
\begin{aligned}
& \frac{\min \left\{\varrho_{i}^{\star}, \sigma_{j}^{\star}\right\} \min _{i \in \ell, j \in \hbar}\left\{\underline{\iota}_{i}, \underline{\vartheta}_{j}\right\}}{\max _{i \in \ell, j \in \hbar}\left\{\bar{\iota}_{i}, \bar{\vartheta}_{j}\right\}} \\
& >\max \left\{\sum_{i=1}^{n} \max _{j \in \hbar} c_{j i}^{*} L_{j}^{f}, \sum_{j=1}^{m} \max _{i \in \ell} d_{i j}^{*} L_{i}^{g}\right\} \\
& \quad+\max \left\{\sum_{i=1}^{n} \max _{j \in \hbar} \alpha_{j i}^{*} L_{j}^{f}, \sum_{i=1}^{n} \max _{j \in \hbar} \beta_{j i}^{*} L_{j}^{f}, \sum_{j=1}^{m} \max _{i \in \ell} p_{i j}^{*} L_{i}^{g}, \sum_{j=1}^{m} \max _{i \in \ell} q_{i j}^{*} L_{i}^{g}\right\} \\
& \quad \times \int_{0}^{\infty} \tilde{K}(s) d s .
\end{aligned}
$$

(H9) There exist constants $M \geq 1, \lambda \in\left(0, \lambda_{0}\right)$ and $\eta \in(0, \lambda)$ such that $\prod_{l=1}^{n} \max \left\{1, \chi_{l}\right\} \leq M e^{\eta t_{n}}$ for all $n \in \mathbb{Z}_{+}$holds and

$$
\begin{aligned}
\lambda< & \frac{\min \left\{\varrho_{i}^{\star}, \sigma_{j}^{\star}\right\} \min _{i \in \ell, j \in \hbar}\left\{\underline{\iota}_{i}, \underline{\vartheta}_{j}\right\}}{\max _{i \in \ell, j \in \hbar}\left\{\bar{l}_{i}, \bar{\vartheta}_{j}\right\}}-\max \left\{\sum_{i=1}^{n} \max _{j \in \hbar} c_{j i}^{*} L_{j}^{f}, \sum_{j=1}^{m} \max _{i \in \ell} d_{i j}^{*} L_{i}^{g}\right\} e^{\lambda \tau} \\
& -\max \left\{\sum_{i=1}^{n} \max _{j \in \hbar} \alpha_{j i}^{*} L_{j}^{f}, \sum_{i=1}^{n} \max _{j \in \hbar} \beta_{j i}^{*} L_{j}^{f}, \sum_{j=1}^{m} \max _{i \in \ell} p_{i j}^{*} L_{i}^{g}, \sum_{j=1}^{m} \max _{i \in \ell} q_{i j}^{*} L_{i}^{g}\right\} \\
& \times \int_{0}^{\infty} \tilde{K}(s) d s,
\end{aligned}
$$

where

$$
\chi_{l}=\frac{\max _{i \in \ell, j \in \hbar}\left\{\bar{l}_{i}, \bar{\vartheta}_{j}\right\}}{\min _{i \in \ell, j \in \hbar}\left\{\underline{\iota}_{i}, \underline{\vartheta}_{j}\right\}} \max _{i \in \ell, j \in \hbar}\left\{\left|1-\gamma_{i l}\right|,\left|1-\delta_{j l}\right|+\max \left\{\sum_{i=1}^{n} \max _{j \in \hbar} e_{i j}^{*} L_{j}^{E}, \sum_{j=1}^{m} \max _{i \in \ell} h_{j i}^{*} L_{i}^{H}\right\} e^{\lambda \tau}\right\} .
$$

Proof Assume that $u(t)=\left(x_{1}\left(t, \phi_{1}\right), x_{2}\left(t, \phi_{1}\right), \ldots, x_{n}\left(t, \phi_{1}\right), y_{1}\left(t, \phi_{2}\right), y_{2}\left(t, \phi_{2}\right), \ldots, y_{m}\left(t, \phi_{2}\right)\right)^{T}$ is an arbitrary solution of system (1.1) through $\left(t, \phi_{1}, \phi_{2}\right)$, where $\phi_{1}=\left(\phi_{11}, \phi_{12}, \ldots, \phi_{1 n}\right)^{T}$, 
$\phi_{2}=\left(\phi_{21}, \phi_{22}, \ldots, \phi_{2 m}\right)^{T}$. Define

$$
x_{i}\left(t+\omega, \phi_{1}\right)=\varphi_{1 i}, \quad t \leq 0, i \in \ell, \quad y_{j}\left(t+\omega, \phi_{2}\right)=\varphi_{2 j}, \quad t \leq 0, j \in \hbar,
$$

then $\varphi_{1}=\left(\varphi_{11}, \varphi_{12}, \ldots, \varphi_{1 n}\right)^{T} \in \mathbb{C}^{n}, \varphi_{2}=\left(\varphi_{21}, \varphi_{22}, \ldots, \varphi_{2 m}\right)^{T} \in \mathbb{C}^{m}$.

Now we construct the following Lyapunov function:

$$
\begin{aligned}
V(t)= & \sum_{i=1}^{n} \int_{x_{i}(t)}^{x_{i}(t+\omega)} \frac{1}{\iota_{i}(s)} d s \operatorname{sgn}\left[x_{i}(t+\omega)-x_{i}(t)\right] \\
& +\sum_{j=1}^{m} \int_{y_{j}(t)}^{y_{j}(t+\omega)} \frac{1}{\vartheta_{j}(s)} d s \operatorname{sgn}\left[y_{j}(t+\omega)-y_{j}(t)\right] .
\end{aligned}
$$

It is easy to see that

$$
\begin{aligned}
& \min _{i \in \ell, j \in \hbar}\left\{\frac{1}{\bar{\iota}_{i}}, \frac{1}{\bar{\vartheta}_{j}}\right\}\left(\sum_{i=1}^{n}\left|x_{i}(t+\omega)-x_{i}(t)\right|+\sum_{j=1}^{m}\left|y_{j}(t+\omega)-y_{j}(t)\right|\right) \\
& \leq V(t) \leq \max _{i \in \ell, j \in \hbar}\left\{\frac{1}{\underline{\iota}_{i}}, \frac{1}{\underline{\vartheta}_{j}}\right\}\left(\sum_{i=1}^{n}\left|x_{i}(t+\omega)-x_{i}(t)\right|+\sum_{j=1}^{m}\left|y_{j}(t+\omega)-y_{j}(t)\right|\right) .
\end{aligned}
$$

When $t \neq t_{k}$, calculating the derivative of $D^{+} V(t)$ along the solution of (1.1), we have

$$
\begin{aligned}
& D^{+} V(t) \leq \sum_{i=1}^{n}\left[\frac{D^{+} x_{i}(t+\omega)}{\iota_{i}\left(x_{i}(t+\omega)\right)}-\frac{D^{+} x_{i}(t)}{\iota_{i}\left(x_{i}(t)\right)}\right] \operatorname{sgn}\left[x_{i}(t+\omega)-x_{i}(t)\right] \\
& +\sum_{j=1}^{m}\left[\frac{D^{+} y_{j}(t+\omega)}{\vartheta_{j}\left(y_{j}(t+\omega)\right)}-\frac{D^{+} y_{j}(t)}{\vartheta_{j}\left(y_{j}(t)\right)}\right] \operatorname{sgn}\left[y_{j}(t+\omega)-y_{j}(t)\right] \\
& =\sum_{i=1}^{n}\left[-a_{i}\left(t, x_{i}(t+\omega)\right)+a_{i}\left(t, x_{i}(t)\right)+\sum_{j=1}^{m} c_{j i}(t) f_{j}\left(y_{j}(t+\omega-\tau(t+\omega))\right)\right. \\
& -\sum_{j=1}^{m} c_{j i}(t) f_{j}\left(y_{j}(t-\tau(t))\right)+\bigwedge_{j=1}^{m} \alpha_{j i}(t) \int_{-\infty}^{t+\omega} K_{j i}(t+\omega-s) f_{j}\left(y_{j}(s)\right) d s \\
& -\bigwedge_{j=1}^{m} \alpha_{j i}(t) \int_{-\infty}^{t} K_{j i}(t-s) f_{j}\left(y_{j}(s)\right) d s+\bigvee_{j=1}^{m} \beta_{j i}(t) \int_{-\infty}^{t+\omega} K_{j i}(t+\omega-s) f_{j}\left(y_{j}(s)\right) d s \\
& \left.-\bigvee_{j=1}^{m} \beta_{j i}(t) \int_{-\infty}^{t} K_{j i}(t-s) f_{j}\left(y_{j}(s)\right) d s\right] \operatorname{sgn}\left[x_{i}(t+\omega)-x_{i}(t)\right] \\
& +\sum_{j=1}^{m}\left[-b_{j}\left(t, y_{j}(t+\omega)\right)+b_{j}\left(t, y_{j}(t)\right)+\sum_{i=1}^{n} d_{i j}(t) g_{i}\left(x_{i}(t+\omega-\tau(t+\omega))\right)\right. \\
& -\sum_{i=1}^{n} d_{i j}(t) g_{i}\left(x_{i}(t-\tau(t))\right)+\bigwedge_{i=1}^{n} p_{i j}(t) \int_{-\infty}^{t+\omega} N_{i j}(t+\omega-s) g_{i}\left(x_{i}(s)\right) d s \\
& -\bigwedge_{i=1}^{n} p_{i j}(t) \int_{-\infty}^{t} N_{i j}(t-s) g_{i}\left(x_{i}(s)\right) d s+\bigvee_{i=1}^{n} q_{i j}(t) \int_{-\infty}^{t+\omega} N_{i j}(t+\omega-s) g_{i}\left(x_{i}(s)\right) d s
\end{aligned}
$$




$$
\begin{aligned}
& \left.-\bigvee_{i=1}^{n} q_{i j}(t) \int_{-\infty}^{t+\omega} N_{i j}(t+\omega-s) g_{i}\left(x_{i}(s)\right) d s\right] \operatorname{sgn}\left[y_{j}(t+\omega)-y_{j}(t)\right] \\
& \leq-\min _{i \in \ell} \varrho_{i}^{\star} \sum_{i=1}^{n}\left|x_{i}(t+\omega)-x_{i}(t)\right|+\sum_{i=1}^{n} \sum_{j=1}^{m} c_{j i}^{*} L_{j}^{f}\left|y_{j}(t+\omega-\tau(t))-y_{j}(t-\tau(t))\right| \\
& +\sum_{i=1}^{n} \sum_{j=1}^{m} \alpha_{j i}^{*} L_{j}^{f} \int_{0}^{\infty} \tilde{K}(s)\left|y_{j}(t+\omega-s)-y_{j}(t-s)\right| d s \\
& +\sum_{i=1}^{n} \sum_{j=1}^{m} \beta_{j i}^{*} L_{j}^{f} \int_{0}^{\infty} \tilde{K}(s)\left|y_{j}(t+\omega-s)-y_{j}(t-s)\right| d s \\
& -\min _{j \in \hbar} \sigma_{j}^{\star} \sum_{j=1}^{m}\left|y_{j}(t+\omega)-y_{j}(t)\right|+\sum_{j=1}^{m} \sum_{i=1}^{n} d_{i j}^{*} L_{i}^{g}\left|x_{i}(t+\omega-\tau(t))-x_{i}(t-\tau(t))\right| \\
& +\sum_{j=1}^{m} \sum_{i=1}^{n} p_{i j}^{*} L_{i}^{g} \int_{0}^{\infty} \tilde{K}(s)\left|x_{i}(t+\omega-s)-x_{i}(t-s)\right| d s \\
& +\sum_{j=1}^{m} \sum_{i=1}^{n} q_{i j}^{*} L_{i}^{g} \int_{0}^{\infty} \tilde{K}(s)\left|x_{i}(t+\omega-s)-x_{i}(t-s)\right| d s \\
& \leq-\min \left\{\varrho_{i}^{\star}, \sigma_{j}^{\star}\right\}\left[\sum_{i=1}^{n}\left(x_{i}(t+\omega)-x_{i}(t)\right)+\sum_{j=1}^{m}\left(y_{j}(t+\omega)-y_{j}(t)\right)\right] \\
& +\sum_{i=1}^{n} \max _{j \in \hbar} c_{j i}^{*} L_{j}^{f} \sum_{j=1}^{m}\left|y_{j}(t+\omega-\tau(t))-y_{j}(t-\tau(t))\right| \\
& +\sum_{i=1}^{n} \max _{j \in \hbar} \alpha_{j i}^{*} L_{j}^{f} \int_{0}^{\infty} \tilde{K}(s)\left|y_{j}(t+\omega-s)-y_{j}(t-s)\right| d s \\
& +\sum_{i=1}^{n} \max _{j \in \hbar} \beta_{j i}^{*} L_{j}^{f} \int_{0}^{\infty} \tilde{K}(s) \sum_{j=1}^{m}\left|y_{j}(t+\omega-s)-y_{j}(t-s)\right| d s \\
& +\sum_{j=1}^{m} \max _{i \in \ell} d_{i j}^{*} L_{i}^{g} \sum_{i=1}^{n}\left|x_{i}(t+\omega-\tau(t))-x_{i}(t-\tau(t))\right| \\
& +\sum_{j=1}^{m} \max _{i \in \ell} p_{i j}^{*} L_{i}^{g} \int_{0}^{\infty} \tilde{K}(s) \sum_{i=1}^{n}\left|x_{i}(t+\omega-s)-x_{i}(t-s)\right| d s \\
& +\sum_{j=1}^{m} \max _{i \in \ell} q_{i j}^{*} L_{i}^{g} \int_{0}^{\infty} \tilde{K}(s) \sum_{i=1}^{n}\left|x_{i}(t+\omega-s)-x_{i}(t-s)\right| d s \\
& \leq-\min \left\{\varrho_{i}^{\star}, \sigma_{j}^{\star}\right\}\left[\sum_{i=1}^{n}\left(x_{i}(t+\omega)-x_{i}(t)\right)+\sum_{j=1}^{m}\left(y_{j}(t+\omega)-y_{j}(t)\right)\right] \\
& +\max \left\{\sum_{i=1}^{n} \max _{j \in \hbar} c_{j i}^{*} L_{j}^{f}, \sum_{j=1}^{m} \max _{i \in \ell} d_{i j}^{*} L_{i}^{g}\right\} \\
& \times\left[\sum_{i=1}^{n}\left|x_{i}(t+\omega-\tau(t))-x_{i}(t-\tau(t))\right|+\sum_{j=1}^{m}\left|y_{j}(t+\omega-\tau(t))-y_{j}(t-\tau(t))\right|\right]
\end{aligned}
$$




$$
\begin{aligned}
& +\max \left\{\sum_{i=1}^{n} \max _{j \in \hbar} \alpha_{j i}^{*} L_{j}^{f}, \sum_{i=1}^{n} \max _{j \in \hbar} \beta_{j i}^{*} L_{j}^{f}, \sum_{j=1}^{m} \max _{i \in \ell} p_{i j}^{*} L_{i}^{g}, \sum_{j=1}^{m} \max _{i \in \ell} q_{i j}^{*} L_{i}^{g}\right\} \\
& \times \int_{0}^{\infty} \tilde{K}(s)\left[\sum_{i=1}^{n}\left|x_{i}(t+\omega-s)-x_{i}(t-s)\right|+\sum_{j=1}^{m}\left|y_{j}(t+\omega-s)-y_{j}(t-s)\right|\right] d s .
\end{aligned}
$$

In view of (3.2), it follows from (3.3) that

$$
\begin{aligned}
D^{+} V(t) \leq & -\min \left\{\varrho_{i}^{\star}, \sigma_{j}^{\star}\right\} \min _{i \in \ell, j \in \hbar}\left\{\underline{\iota}_{i}, \underline{\vartheta}_{j}\right\} V(t) \\
& +\max \left\{\sum_{i=1}^{n} \max _{j \in \hbar} c_{j i}^{*} L_{j}^{f}, \sum_{j=1}^{m} \max _{i \in \ell} d_{i j}^{*} L_{i}^{g}\right\} \max _{i \in \ell, j \in \hbar}\left\{\bar{\iota}_{i}, \bar{\vartheta}_{j}\right\} V(t-\tau(t)) \\
& +\max \left\{\sum_{i=1}^{n} \max _{j \in \hbar} \alpha_{j i}^{*} L_{j}^{f}, \sum_{i=1}^{n} \max _{j \in \hbar} \beta_{j i}^{*} L_{j}^{f}, \sum_{j=1}^{m} \max _{i \in \ell} p_{i j}^{*} L_{i}^{g}, \sum_{j=1}^{m} \max _{i \in \ell} q_{i j}^{*} L_{i}^{g}\right\} \\
& \times \int_{0}^{\infty} \tilde{K}(s) V(t-s) d s .
\end{aligned}
$$

When $t=t_{k}$, in view of (H6), (H7), and (3.2), we get

$$
\begin{aligned}
& V\left(t_{k}\right) \leq \max _{i \in \ell, j \in \hbar}\left\{\frac{1}{\underline{\iota}_{i}}, \frac{1}{\underline{\vartheta}_{j}}\right\}\left(\sum_{i=1}^{n}\left|x_{i}\left(t_{k}+\omega\right)-x_{i}\left(t_{k}\right)\right|+\sum_{j=1}^{m}\left|y_{j}\left(t_{k}+\omega\right)-y_{j}\left(t_{k}\right)\right|\right) \\
& =\max _{i \in \ell, j \in \hbar}\left\{\frac{1}{\underline{\iota}_{i}}, \frac{1}{\underline{\vartheta}_{j}}\right\}\left(\sum_{i=1}^{n}\left|x_{i}\left(t_{k+q}\right)-x_{i}\left(t_{k}\right)\right|+\sum_{j=1}^{m}\left|y_{j}\left(t_{k+q}\right)-y_{j}\left(t_{k}\right)\right|\right) \\
& \leq \max _{i \in \ell, j \in \hbar}\left\{\frac{1}{\underline{\iota}_{i}}, \frac{1}{\underline{\vartheta}_{j}}\right\}\left\{\sum_{i=1}^{n}\left|1-\gamma_{i k}\right|\left|x_{i}\left(t_{k+q}^{-}\right)-x_{i}\left(t_{k}^{-}\right)\right|\right. \\
& +\sum_{i=1}^{n} \sum_{j=1}^{m} e_{i j}^{*} L_{j}^{E}\left|y_{j}\left(t_{k+q}^{-}-\tau\right)-y_{j}\left(t_{k}^{-}-\tau\right)\right| \\
& \left.+\sum_{j=1}^{m}\left|1-\delta_{j k}\right|\left|y_{j}\left(t_{k+q}^{-}\right)-y_{j}\left(t_{k}^{-}\right)\right|+\sum_{j=1}^{m} \sum_{i=1}^{n} h_{j i}^{*} L_{i}^{H}\left|x_{i}\left(t_{k+q}^{-}-\tau\right)-x_{i}\left(t_{k}^{-}-\tau\right)\right|\right\} \\
& \leq \max _{i \in \ell, j \in \hbar}\left\{\frac{1}{\underline{\iota}_{i}}, \frac{1}{\underline{\vartheta}_{j}}\right\}\left\{\max _{i \in \ell}\left|1-\gamma_{i k}\right| \sum_{i=1}^{n}\left|x_{i}\left(t_{k}^{-}+\omega\right)-x_{i}\left(t_{k}^{-}\right)\right|\right. \\
& +\sum_{i=1}^{n} \max _{j \in \hbar} e_{i j}^{*} L_{j}^{E} \sum_{j=1}^{m}\left|y_{j}\left(t_{k}^{-}+\omega-\tau\right)-y_{j}\left(t_{k}^{-}-\tau\right)\right| \\
& +\max _{j \in \hbar}\left|1-\delta_{j k}\right| \sum_{j=1}^{m}\left|y_{j}\left(t_{k}^{-}+\omega\right)-y_{j}\left(t_{k}^{-}\right)\right| \\
& \left.+\sum_{i=1}^{n} \max _{i \in \ell} h_{j i}^{*} L_{i}^{H} \sum_{j=1}^{m}\left|x_{i}\left(t_{k}^{-}+\omega-\tau\right)-x_{i}\left(t_{k}^{-}-\tau\right)\right|\right\} \\
& \leq \max _{i \in \ell, j \in \hbar}\left\{\frac{1}{\underline{\iota}_{i}}, \frac{1}{\underline{\vartheta}_{j}}\right\} \max _{i \in \ell, j \in \hbar}\left\{\left|1-\gamma_{i k}\right|,\left|1-\delta_{j k}\right|\right\}
\end{aligned}
$$




$$
\begin{aligned}
& \times\left(\sum_{i=1}^{n}\left|x_{i}\left(t_{k}^{-}+\omega\right)-x_{i}\left(t_{k}^{-}\right)\right|+\sum_{j=1}^{m}\left|y_{j}\left(t_{k}^{-}+\omega\right)-y_{j}\left(t_{k}^{-}\right)\right|\right) \\
& +\max \left\{\sum_{i=1}^{n} \max _{j \in \hbar} e_{i j}^{*} L_{j}^{E}, \sum_{i=1}^{n} \max _{i \in \ell} h_{j i}^{*} L_{i}^{H}\right\} \\
& \times\left(\sum_{i=1}^{n}\left|x_{i}\left(t_{k}^{-}+\omega\right)-x_{i}\left(t_{k}^{-}\right)\right|+\sum_{j=1}^{m}\left|y_{j}\left(t_{k}^{-}+\omega\right)-y_{j}\left(t_{k}^{-}\right)\right|\right) \\
& \leq \frac{\max _{i \in \ell, j \in \hbar}\left\{\bar{\imath}_{i}, \bar{\vartheta}_{j}\right\}}{\min _{i \in \ell, j \in \hbar}\left\{\underline{\iota}_{i}, \underline{\vartheta}_{j}\right\}} \max _{i \in \ell, j \in \hbar}\left\{\left|1-\gamma_{i k}\right|,\left|1-\delta_{j k}\right|\right\} V\left(t_{k}^{-}\right) \\
& +\frac{\max _{i \in \ell, j \in \hbar}\left\{\bar{l}_{i}, \bar{\vartheta}_{j}\right\}}{\min _{i \in \ell, j \in \hbar}\left\{\underline{\iota}_{i}, \underline{\vartheta}_{j}\right\}} \max \left\{\sum_{i=1}^{n} \max _{j \in \hbar} e_{i j}^{*} L_{j}^{E}, \sum_{i=1}^{n} \max _{i \in \ell} h_{j i}^{*} L_{i}^{H}\right\} V\left(t_{k}^{-}-\tau\right) .
\end{aligned}
$$

In view of (3.4)-(3.5) and (H8)-(H9), using Lemma 2.2, we have

$$
V(t) \leq M \bar{V}(0) e^{-(\lambda-\eta) t}, \quad t \geq 0
$$

where $\bar{V}(0)=\sup _{-\infty \leq s \leq 0} V(s)$. It follows from (3.6) that

$$
\sum_{i=1}^{n}\left|x_{i}(t+\omega)-x_{i}(t)\right|+\sum_{j=1}^{m}\left|y_{j}(t+\omega)-y_{j}(t)\right| \leq v\|\phi-\varphi\| e^{-(\lambda-\eta) t}, \quad t \geq 0
$$

where

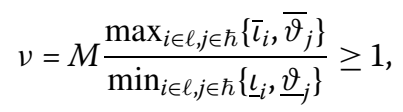

$\lambda$ satisfies the condition (H9). Notice that

$$
\begin{aligned}
& x_{i}(t+k \omega)=x_{i}(t)+\sum_{l=1}^{k}\left[x_{i}(t+l \omega)-x_{i}(t+(l-1) \omega)\right], \quad i \in \ell, \\
& y_{j}(t+k \omega)=y_{j}(t)+\sum_{l=1}^{k}\left[y_{j}(t+l \omega)-y_{j}(t+(l-1) \omega)\right], \quad j \in \hbar .
\end{aligned}
$$

In view of (3.7), we have

$$
\begin{aligned}
& \sum_{l=1}^{\infty}\left[x_{i}(t+l \omega)-x_{i}(t+(l-1) \omega)\right] \\
& \quad=\lim _{k \rightarrow \infty} \sum_{l=1}^{k}\left[x_{i}(t+l \omega)-x_{i}(t+(l-1) \omega)\right] \\
& \quad \leq v\|\phi-\varphi\| \lim _{k \rightarrow \infty} \sum_{l=1}^{k} e^{-(\lambda-\eta)(t+(l-1) \omega)} \\
& \quad \leq v\|\phi-\varphi\| e^{-(\lambda-\eta) t} \sum_{l=1}^{\infty} e^{-(\lambda-\eta)(l-1) \omega}<\infty, \quad \text { as } k \rightarrow \infty,
\end{aligned}
$$


for any given $t \geq 0$. By (3.8), we know that $\lim _{k \rightarrow \infty} x_{i}(t+k \omega)$ exists. Similarly, we know that $\lim _{k \rightarrow \infty} y_{j}(t+k \omega)$ also exists.

Set $\left(x^{*}(t), y^{*}(t)\right)^{T}=\left(x_{1}^{*}(t), x_{2}^{*}(t), \ldots, x_{n}^{*}(t), y_{1}^{*}(t), y_{2}^{*}(t), \ldots, y_{m}^{*}(t)\right)^{T}$, where $x_{i}^{*}=\lim _{k \rightarrow \infty} x_{i}(t+$ $k \omega), y_{j}^{*}=\lim _{k \rightarrow \infty} y_{j}(t+k \omega)$, then $\left(x^{*}(t), y^{*}(t)\right)^{T}$ is a periodic function with period $\omega$ for system (1.1).

Assume that system (1.1) has another $\omega$-periodic solution $\left(x^{* *}(t), y^{* *}(t)\right)^{T}$ as follows:

$$
\begin{aligned}
\left(x^{* *}\left(t, \psi_{1}\right), y^{* *}\left(t, \psi_{2}\right)\right)^{T}= & \left(x_{1}^{* *}\left(t, \psi_{1}\right), x_{2}^{* *}\left(t, \psi_{1}\right), \ldots, x_{n}^{* *}\left(t, \psi_{1}\right),\right. \\
& \left.y_{1}^{* *}\left(t, \psi_{2}\right), y_{2}^{* *}\left(t, \psi_{2}\right), \ldots, y_{m}^{* *}\left(t, \psi_{2}\right)\right)^{T},
\end{aligned}
$$

where $\psi_{1} \in \mathbb{C}^{n}, \psi_{2} \in \mathbb{C}^{m}$. It follows from (3.7) that

$$
\begin{aligned}
& \sum_{i=1}^{n}\left|x_{i}^{*}(t)-x_{i}^{* *}(t)\right|+\sum_{j=1}^{m}\left|y_{j}^{*}(t)-y_{j}^{* *}(t)\right| \\
& \quad=\sum_{i=1}^{n}\left|x_{i}^{*}(t+k \omega)-x_{i}^{* *}(t+k \omega)\right|+\sum_{j=1}^{m}\left|y_{j}^{*}(t+k \omega)-y_{j}^{* *}(t+k \omega)\right| \\
& \quad \leq v\|\phi-\psi\| e^{(\lambda-\eta)(t+k \omega)}, \quad t \geq 0 .
\end{aligned}
$$

Let $k \rightarrow \infty$, then $x_{i}^{*}(t)=x_{i}^{* *}(t), y_{j}^{*}(t)=y_{j}^{* *}(t), t \geq 0$. Thus we can conclude that system (1.1) has a unique $\omega$-periodic solution which is globally exponentially stable. The proof of Theorem 3.1 is complete.

Remark 3.1 Li [4] investigated the existence and global exponential stability of a periodic solution for impulsive Cohen-Grossberg-type BAM neural networks with continuously distributed delays, the model in [4] is not concerned with fuzzy terms. Bao [10] discussed the existence and exponential stability of a periodic solution for BAM fuzzy CohenGrossberg neural networks with mixed delays, the model in [10] is not concerned with impulsive effects. Yang [16] considered the periodic solution for fuzzy Cohen-Grossberg BAM neural networks with both time-varying and distributed delays and variable coefficients, the model in [16] is not concerned with impulsive effect and distributed delays. Balasubramaniam et al. [38] analyzed the global asymptotic stability of stochastic fuzzy cellular neural networks with multiple time-varying delays, the model in [38] is not concerned with impulsive effect and distributed delays, Balasubramaniam and Vembarasan [41] studied the robust stability of uncertain fuzzy BAM neural networks of neutral-type with Markovian jumping parameters and impulses, the authors did not discuss the existence and global exponential stability of a periodic solution of neural networks and the model in [41] is also not concerned with distributed delays. In this paper, we study the exponential stability for fuzzy bidirectional associative memory Cohen-Grossberg neural networks with mixed delays and impulses. All the obtained results in $[4,10,16,38$, 41] cannot be applicable to model (1.1) to obtain the exponential stability of model (1.1). From this viewpoint, our results on the exponential stability for fuzzy bidirectional associative memory Cohen-Grossberg neural networks with mixed delays and impulses are essentially new and complement earlier works to some extent. 


\section{Examples}

In this section, we consider the following neural networks with mixed delays and impulses

$$
\left\{\begin{aligned}
\dot{x}_{1}(t)= & l_{1}\left(x_{1}(t)\right)\left[-a_{1}\left(t, x_{1}(t)\right)+\sum_{j=1}^{2} c_{j 1}(t) f_{j}\left(y_{j}(t-\tau(t))\right)\right. \\
& +\bigwedge_{j=1}^{2} \alpha_{j 1}(t) \int_{-\infty}^{t} K_{j 1}(t-s) f_{j}\left(y_{j}(s)\right) d s+\bigwedge_{j=1}^{2} T_{j 1} u_{j}+\bigvee_{j=1}^{2} H_{j 1} u_{j} \\
& \left.+\bigvee_{j=1}^{2} \beta_{j 1}(t) \int_{-\infty}^{t} K_{j 1}(t-s) f_{j}\left(y_{j}(s)\right) d s+I_{1}(t)\right], \quad t \neq t_{k}, \\
\dot{x}_{2}(t)= & l_{2}\left(x_{2}(t)\right)\left[-a_{2}\left(t, x_{2}(t)\right)+\sum_{j=1}^{2} c_{j 2}(t) f_{j}\left(y_{j}(t-\tau(t))\right)\right. \\
& +\bigwedge_{j=1}^{2} \alpha_{j 2}(t) \int_{-\infty}^{t} K_{j 2}(t-s) f_{j}\left(y_{j}(s)\right) d s+\bigwedge_{j=1}^{2} T_{j 2} u_{j}+\bigvee_{j=1}^{2} H_{j 2} u_{j} \\
& \left.+\bigvee_{j=1}^{2} \beta_{j 2}(t) \int_{-\infty}^{t} K_{j 2}(t-s) f_{j}\left(y_{j}(s)\right) d s+I_{2}(t)\right], \quad t \neq t_{k}, \\
\triangle x_{1}\left(t_{k}\right) & =x_{1}\left(t_{k}\right)-x_{1}\left(t_{k}^{-}\right)=-\gamma_{1 k} x_{i}\left(t_{k}^{-}\right)+\sum_{j=1}^{2} e_{1 j}\left(t_{k}^{-}\right) E_{j}\left(y_{j}\left(t_{k}^{-}-\tau\right)\right), \quad k \in \mathbb{Z}_{+}, \\
\triangle x_{2}\left(t_{k}\right) & =x_{2}\left(t_{k}\right)-x_{2}\left(t_{k}^{-}\right)=-\gamma_{2 k} x_{2}\left(t_{k}^{-}\right)+\sum_{j=1}^{2} e_{2 j}\left(t_{k}^{-}\right) E_{j}\left(y_{j}\left(t_{k}^{-}-\tau\right)\right), \quad k \in \mathbb{Z}_{+}, \\
\dot{y}_{1}(t)= & \vartheta_{1}\left(y_{1}(t)\right)\left[-b_{1}\left(t, y_{j}(t)\right)+\sum_{i=1}^{2} d_{i 1}(t) g_{i}\left(x_{i}(t-\tau(t))\right)\right. \\
& +\bigwedge_{i=1}^{2} p_{i 1}(t) \int_{-\infty}^{t} N_{i 1}(t-s) g_{i}\left(x_{i}(s)\right) d s+\bigwedge_{i=1}^{2} S_{i 1} u_{i}+\bigvee_{i=1}^{2} L_{i 1} u_{i} \\
& \left.+\bigvee_{i=1}^{2} q_{i 1}(t) \int_{-\infty}^{t} N_{i 1}(t-s) g_{i}\left(x_{i}(s)\right) d s+J_{1}(t)\right], \quad t \neq t_{k}, \\
\dot{y}_{2}(t)= & \vartheta_{2}\left(y_{2}(t)\right)\left[-b_{2}\left(t, y_{j}(t)\right)+\sum_{i=1}^{2} d_{i 2}(t) g_{i}\left(x_{i}(t-\tau(t))\right)\right. \\
& +\bigwedge_{i=1}^{2} p_{i 2}(t) \int_{-\infty}^{t} N_{i 2}(t-s) g_{i}\left(x_{i}(s)\right) d s+\bigwedge_{i=1}^{2} S_{i 2} u_{i}+\bigvee_{i=1}^{2} L_{i 2} u_{i} \\
& \left.+\bigvee_{i=1}^{2} q_{i 2}(t) \int_{-\infty}^{t} N_{i 2}(t-s) g_{i}\left(x_{i}(s)\right) d s+J_{2}(t)\right], \quad t \neq t_{k}, \\
\triangle y_{1}\left(t_{k}\right) & =y_{1}\left(t_{k}\right)-y_{1}\left(t_{k}^{-}\right)=-\delta_{1 k} y_{1}\left(t_{k}^{-}\right)+\sum_{i=1}^{2} h_{1 i}\left(t_{k}^{-}\right) H_{i}\left(x_{i}\left(t_{k}^{-}-\tau\right)\right), \quad k \in \mathbb{Z}_{+}, \\
\triangle y_{2}\left(t_{k}\right) & =y_{2}\left(t_{k}\right)-y_{2}\left(t_{k}^{-}\right)=-\delta_{2 k} y_{2}\left(t_{k}^{-}\right)+\sum_{i=1}^{2} h_{2 i}\left(t_{k}^{-}\right) H_{i}\left(x_{i}\left(t_{k}^{-}-\tau\right)\right), \quad k \in \mathbb{Z}_{+},
\end{aligned}\right.
$$

where

$$
\begin{aligned}
& {\left[\begin{array}{ll}
c_{11}(t) & c_{21}(t) \\
c_{21}(t) & c_{22}(t)
\end{array}\right]=\left[\begin{array}{ll}
0.9+0.2 \sin t & 0.5+0.4 \cos t \\
0.4+0.2 \sin t & 0.5+0.1 \cos t
\end{array}\right],} \\
& {\left[\begin{array}{ll}
d_{11}(t) & d_{21}(t) \\
d_{12}(t) & d_{22}(t)
\end{array}\right]=\left[\begin{array}{ll}
0.5+0.2 \sin t & 0.4+0.2 \cos t \\
0.3+0.1 \sin t & 0.5+0.2 \cos t
\end{array}\right],} \\
& {\left[\begin{array}{ll}
\alpha_{11}(t) & \alpha_{21}(t) \\
\alpha_{12}(t) & \alpha_{22}(t)
\end{array}\right]=\left[\begin{array}{ll}
0.5+0.2 \cos t & 0.5+0.2 \sin t \\
0.4+0.2 \cos t & 0.4+0.2 \sin t
\end{array}\right],} \\
& {\left[\begin{array}{ll}
p_{11}(t) & p_{21}(t) \\
p_{12}(t) & p_{22}(t)
\end{array}\right]=\left[\begin{array}{ll}
0.5+0.2 \cos t & 0.4+0.2 \sin t \\
0.3+0.1 \cos t & 0.3+0.2 \sin t
\end{array}\right],} \\
& {\left[\begin{array}{ll}
\beta_{11}(t) & \beta_{21}(t) \\
\beta_{12}(t) & \beta_{22}(t)
\end{array}\right]=\left[\begin{array}{ll}
0.4+0.1 \sin t & 0.6+0.3 \cos t \\
0.3+0.2 \sin t & 0.5+0.3 \cos t
\end{array}\right],} \\
& {\left[\begin{array}{ll}
q_{11}(t) & q_{21}(t) \\
q_{12}(t) & q_{22}(t)
\end{array}\right]=\left[\begin{array}{ll}
0.5+0.3 \cos t & 0.4+0.1 \sin t \\
0.3+0.2 \cos t & 0.5+0.2 \sin t
\end{array}\right],} \\
& {\left[\begin{array}{ll}
T_{11} & T_{21} \\
T_{12} & t_{22}
\end{array}\right]=\left[\begin{array}{ll}
1 & 1 \\
1 & 1
\end{array}\right], \quad\left[\begin{array}{ll}
S_{11} & S_{21} \\
S_{12} & S_{22}
\end{array}\right]=\left[\begin{array}{ll}
1 & 1 \\
1 & 1
\end{array}\right],} \\
& {\left[\begin{array}{ll}
H_{11} & H_{21} \\
H_{12} & H_{22}
\end{array}\right]=\left[\begin{array}{ll}
1 & 1 \\
1 & 1
\end{array}\right], \quad\left[\begin{array}{ll}
L_{11} & L_{21} \\
L_{12} & L_{22}
\end{array}\right]=\left[\begin{array}{ll}
1 & 1 \\
1 & 1
\end{array}\right],} \\
& {\left[\begin{array}{ll}
u_{1} & u_{2} \\
I_{1} & J_{1}
\end{array}\right]=\left[\begin{array}{ll}
1+\sin t & 1+\cos t \\
1+\sin t & 1+\cos t
\end{array}\right],}
\end{aligned}
$$




$$
\begin{aligned}
& {\left[\begin{array}{ll}
e_{11}(t) & e_{12}(t) \\
e_{21}(t) & e_{22}(t)
\end{array}\right]=\left[\begin{array}{ll}
\tanh (0.2 t) & \tanh (0.2 t) \\
\tanh (0.2 t) & \tanh (0.2 t)
\end{array}\right],} \\
& {\left[\begin{array}{ll}
h_{11}(t) & h_{12}(t) \\
h_{21}(t) & h_{22}(t)
\end{array}\right]=\left[\begin{array}{ll}
\tanh (0.3 t) & \tanh (0.3 t) \\
\tanh (0.3 t) & \tanh (0.3 t)
\end{array}\right], \quad\left[\begin{array}{ll}
\gamma_{1 k}(s) & \gamma_{2 k}(s) \\
\delta_{1 k}(s) & \delta_{1 k}(s)
\end{array}\right]=\left[\begin{array}{ll}
-1 & -1 \\
-1 & -1
\end{array}\right],} \\
& {\left[\begin{array}{cc}
\iota_{1}\left(x_{1}(t)\right) & \vartheta_{1}\left(y_{1}(t)\right) \\
a_{1}\left(t, x_{1}(t)\right) & b_{1}\left(t, y_{1}(t)\right)
\end{array}\right]=\left[\begin{array}{ll}
0.55+0.05 \cos \left(x_{1}(t)\right) & 4 x_{1}(t) \\
0.55+0.05 \sin \left(y_{1}(t)\right) & 3 y_{1}(t)
\end{array}\right],} \\
& {\left[\begin{array}{cc}
\iota_{2}\left(x_{2}(t)\right) & \vartheta_{2}\left(y_{2}(t)\right) \\
a_{2}\left(t, x_{2}(t)\right) & b_{2}\left(t, y_{2}(t)\right)
\end{array}\right]=\left[\begin{array}{ll}
0.55+0.05 \sin \left(x_{2}(t)\right) & 4 x_{2}(t) \\
0.55+0.05 \cos \left(y_{2}(t)\right) & 3 y_{2}(t)
\end{array}\right],} \\
& {\left[\begin{array}{ll}
K_{11}(s) & K_{21}(s) \\
K_{12}(s) & K_{22}(s)
\end{array}\right]=\left[\begin{array}{cc}
e^{-3 s} & e^{-3 s} \\
e^{-3 s} & e^{-3 s}
\end{array}\right], \quad\left[\begin{array}{ll}
N_{11}(s) & N_{21}(s) \\
N_{12}(s) & N_{22}(s)
\end{array}\right]=\left[\begin{array}{ll}
e^{-3 s} & e^{-3 s} \\
e^{-3 s} & e^{-3 s}
\end{array}\right],} \\
& {\left[\begin{array}{ll}
E_{1}\left(y_{1}\left(t_{k}^{-}-\tau\right)\right) & E_{2}\left(y_{2}\left(t_{k}^{-}-\tau\right)\right) \\
H_{1}\left(x_{1}\left(t_{k}^{-}-\tau\right)\right) & H_{2}\left(x_{2}\left(t_{k}^{-}-\tau\right)\right)
\end{array}\right]=\left[\begin{array}{ll}
\tanh \left(0.2 y_{1}\left(t_{k}^{-}-0.2\right)\right) & \tanh \left(0.2 y_{2}\left(t_{k}^{-}-0.2\right)\right) \\
\tanh \left(0.2 x_{1}\left(t_{k}^{-}-0.2\right)\right) & \tanh \left(0.2 x_{2}\left(t_{k}^{-}-0.2\right)\right)
\end{array}\right] .}
\end{aligned}
$$

Let $t_{k}=0.5 \pi k, \tau(t)=0.2|\sin 2 t|, f_{1}(u)=|u+1|, g_{1}(u)=|u-1|$, then we get $\bar{l}_{1}=0.6, \underline{\iota}_{1}=$ $0.5, \bar{\vartheta}_{1}=0.6, \underline{\vartheta}_{1}=0.5, \bar{\iota}_{2}=0.6, \underline{\iota}_{2}=0.5, \bar{\vartheta}_{2}=0.6, \underline{\vartheta}_{2}=0.5, c_{11}^{*}=1.1, c_{21}^{*}=0.9, c_{12}^{*}=0.6$, $c_{22}^{*}=0.6, d_{11}^{*}=0.6, d_{21}^{*}=0.6, d_{12}^{*}=0.4, d_{22}^{*}=0.6, \alpha_{11}^{*}=0.7, \alpha_{21}^{*}=0.7, \alpha_{12}^{*}=0.6, \alpha_{22}^{*}=$ $0.6, p_{11}^{*}=0.6, p_{21}^{*}=0.6, p_{12}^{*}=0.4, p_{22}^{*}=0.5, \beta_{11}^{*}=0.6, \beta_{21}^{*}=0.7, \beta_{12}^{*}=0.5, \beta_{22}^{*}=0.8, q_{11}^{*}=$ $0.4, q_{21}^{*}=0.9, q_{12}^{*}=0.5, q_{22}^{*}=0.7, h_{11}^{*}=1, h_{21}^{*}=1, h_{12}^{*}=1, h_{22}^{*}=1, \tau=0.2, \bar{K}(s)=e^{-3 s}$, $\lambda_{0}=1.5, \omega=2 \pi, L_{1}^{f}=L_{1}^{g}=1, L_{1}^{E}=0.2, L_{2}^{E}=0.2, L_{1}^{H}=0.2, L_{2}^{H}=0.2$. It is easy to check that

$$
\begin{aligned}
& \frac{\min _{i \in \ell, j \in \hbar}\left\{\varrho_{i}^{\star}, \sigma_{j}^{\star}\right\} \min _{i \in \ell, j \in \hbar}\left\{\underline{L}_{i}, \underline{\vartheta}_{j}\right\}}{\max _{i \in \ell, j \in \hbar}\left\{\bar{l}_{i}, \bar{\vartheta}_{j}\right\}}=7.5, \\
& \max \left\{\sum_{i=1}^{n} \max _{j \in \hbar} c_{j i}^{*} L_{j}^{f}, \sum_{j=1}^{m} \max _{i \in \ell} d_{i j}^{*} L_{i}^{g}\right\} \\
& \quad+\max \left\{\sum_{i=1}^{n} \max _{j \in \hbar} \alpha_{j i}^{*} L_{j}^{f}, \sum_{i=1}^{n} \max _{j \in \hbar} \beta_{j i}^{*} L_{j}^{f}, \sum_{j=1}^{m} \max _{i \in \ell} p_{i j}^{*} L_{i}^{g}, \sum_{j=1}^{m} \max _{i \in \ell} q_{i j}^{*} L_{i}^{g}\right\} \int_{0}^{\infty} \tilde{K}(s) d s
\end{aligned}
$$$$
\approx 6.4237 \text {. }
$$

Choose $\lambda=0.9<\lambda_{0}$ such that

$$
\lambda<7.5-2 e^{0.2 \lambda}-1.4 \int_{0}^{\infty} e^{(\lambda-3) s} d s .
$$

Then we obtain

$$
\begin{aligned}
\chi_{l}= & \frac{\max _{i \in \ell, j \in \hbar}\left\{\bar{\imath}_{i}, \bar{\vartheta}_{j}\right\}}{\min _{i \in \ell, j \in \hbar}\left\{\underline{\vartheta}_{i}, \underline{\vartheta}_{j}\right\}} \\
& \times \max _{i \in \ell, j \in \hbar}\left\{\left|1-\gamma_{i l}\right|,\left|1-\delta_{j l}\right|+\max \left\{\sum_{i=1}^{n} \max _{j \in \hbar} e_{i j}^{*} L_{j}^{E}, \sum_{j=1}^{m} \max _{i \in \ell} h_{j i}^{*} L_{i}^{H}\right\} e^{\lambda \tau}\right\} \\
\approx & 1.9503 .
\end{aligned}
$$




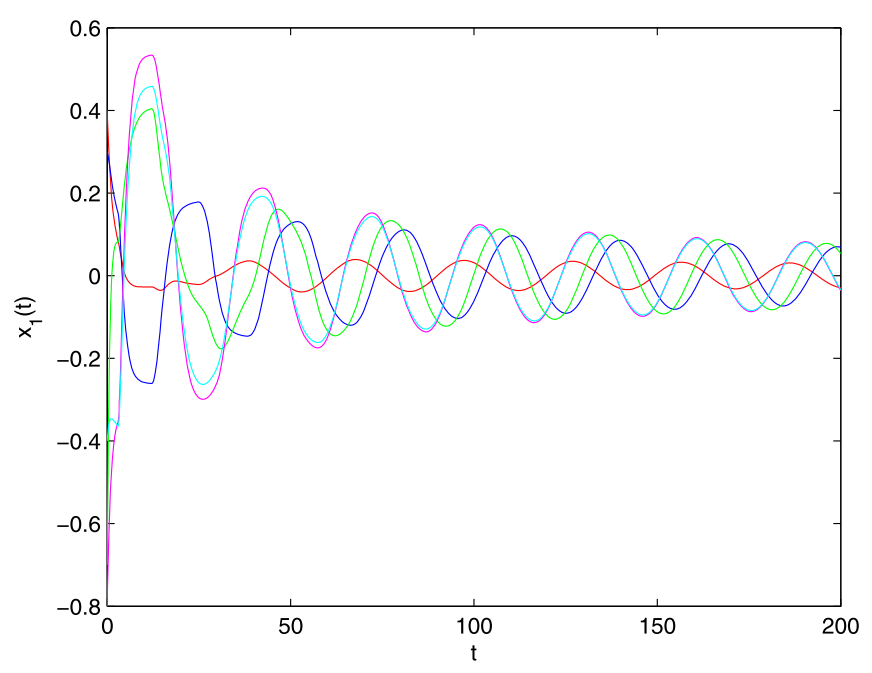

Figure 1 Numerical solutions of system (3.1): times series of $x_{1}$.

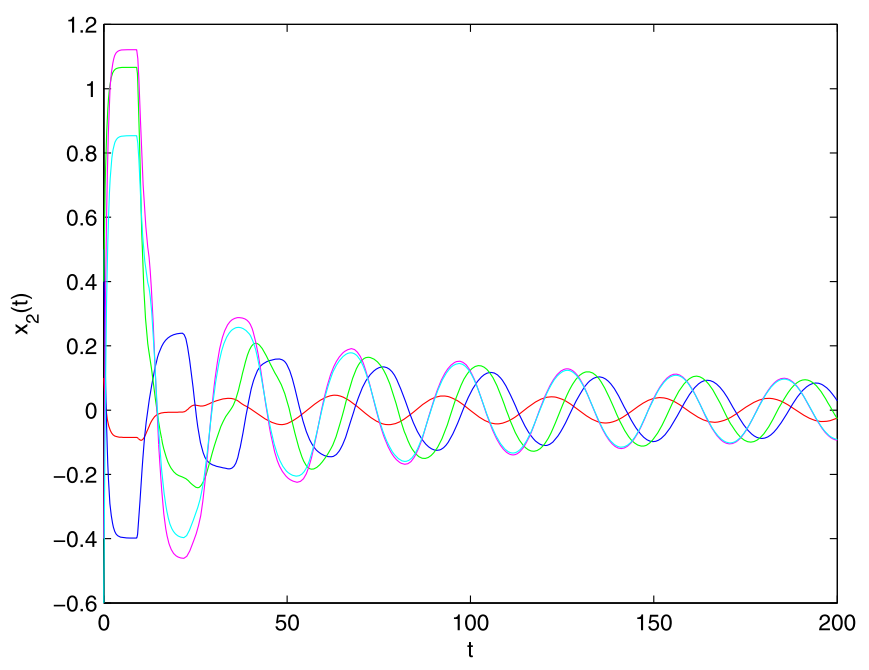

Figure 2 Numerical solutions of system (3.1): times series of $x_{2}$.

Thus we can choose $\eta=0.95<\lambda$ such that $\prod_{l=1}^{n} \max \left\{1, \chi_{l}\right\}=1.9503^{n}<2.5857^{n} \approx e^{\eta t_{n}}$ for all $n \in \mathbb{Z}_{+}$. Then all the conditions of Theorem 3.1 hold. Thus (4.1) has exactly one $2 \pi$ periodic solution which is globally exponentially stable. These results are illustrated in Figures 1, 2, 3, 4.

\section{Conclusions}

In this article, we have analyzed the global exponential stability of fuzzy bidirectional associative memory Cohen-Grossberg neural networks with mixed delays and impulses. By constructing a suitable Lyapunov function and a new differential inequality, some sufficient criteria which ensure the existence and global exponential stability of a periodic solution of the model have been established. The obtained conditions are easy to check in practice. The results in this paper extend and complement some previous studies. Finally, 


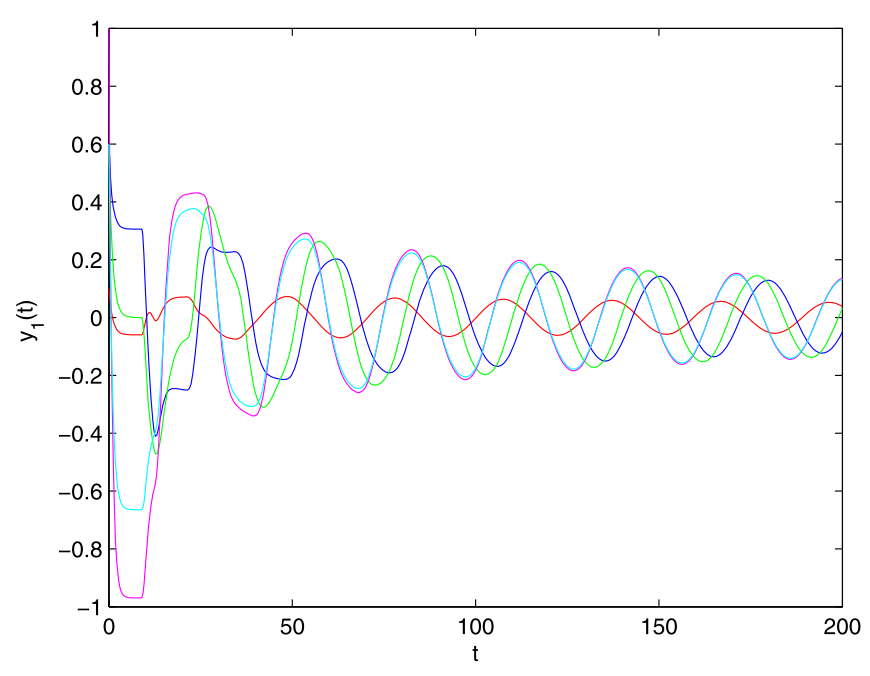

Figure 3 Numerical solutions of system (3.1): times series of $y_{1}$.

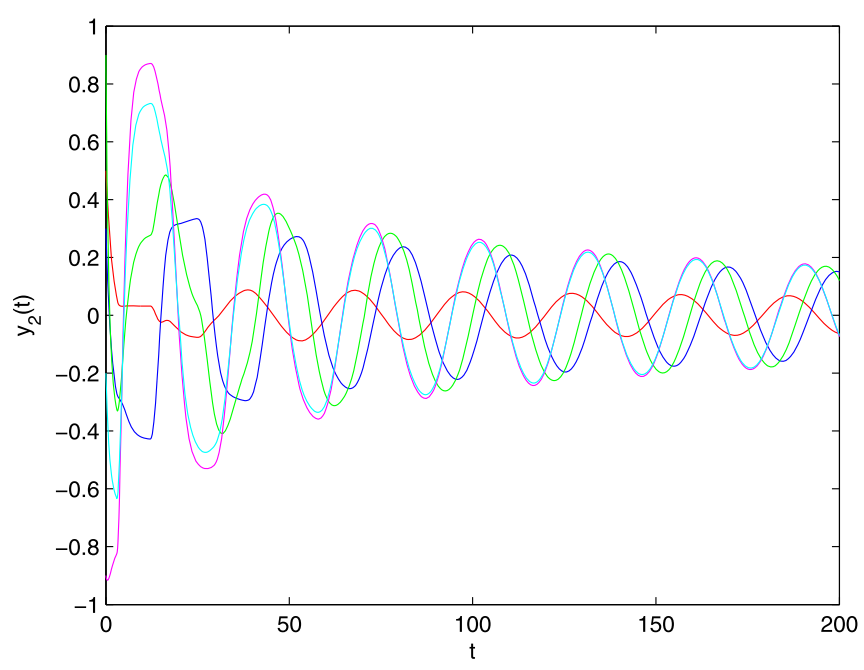

Figure 4 Numerical solutions of system (3.1): times series of $y_{2}$.

an example with their numerical simulations is carried out to illustrate the correctness. To the best of our knowledge, there are only rare results on the exponential stability for fuzzy bidirectional associative memory Cohen-Grossberg neural networks with proportional delays, which will be our future research direction.

Competing interests

The authors declare that there is no conflict of interest regarding the publication of this paper.

Authors' contributions

The authors have equally made contributions. All authors read and approved the final manuscript.

\section{Acknowledgements}

The authors would like to thank the referees and the editor for helpful suggestions incorporated into this paper.

Received: 10 September 2016 Accepted: 4 January 2017 Published online: 21 February 2017 


\section{References}

1. Cohen, MA, Grossberg, S: Absolute stability of global pattern formation and parallel memory storage by competitive neural networks. IEEE Trans. Syst. Man Cybern. 13, 815-826 (1983)

2. Kang, HY, Fu, XC, Sun, ZL: Global exponential stability of a periodic solutions for impulsive Cohen-Grossberg neural networks with delays. Appl. Math. Model. 39, 1526-1535 (2015)

3. Wu, HX, Liao, XF, Feng, W, Guo, ST: Mean square stability of uncertain stochastic BAM neural networks with interval time-varying delays. Cogn. Neurodyn. 6, 443-458 (2012)

4. Li, XD: Existence and global exponential stability of periodic solution for impulsive Cohen-Grossberg-type BAM neural networks with continuously distributed delays. Appl. Math. Comput. 215, 292-307 (2009)

5. Hasan, S, Siong, N: A parallel processing VLSI BAM engine. IEEE Trans. Neural Netw. 8, 424-436 (1997)

6. Teddy, SD, Ng, SK: Forecasting ATM cash demands using a local learning model of cerebellar associative memory network. Int. J. Forecast. 27, 760-776 (2011)

7. Chang, H, Feng, Z, Wei, X: Object recognition and tracking with maximum likelihood bidirectional associative memory networks. Neurocomputing 72, 278-292 (2008)

8. Sudo, A, Sato, A, Hasegawa, O: Associative memory for online learning in noisy environments using self-organizing incremental neural network. IEEE Trans. Neural Netw. 20, 964-972 (2009)

9. Zhou, LQ, Chen, XB, Yang, YX: Asymptotic stability of cellular neural networks with multiple proportional delays. Appl. Math. Comput. 229, 457-466 (2014)

10. Bao, HM: Existence and exponential stability of periodic solution for BAM fuzzy Cohen-Grossberg neural networks with mixed delays. Neural Process. Lett. 43, 871-885 (2016)

11. Sakthivel, R, Arunkumar, A, Mathiyalagan, K, Marshal Anthoni, S: Robust passivity analysis of fuzzy Cohen-Grossberg BAM neural networks with time-varying delays. Appl. Math. Comput. 218, 3799-3809 (2011)

12. Zhang, ZQ, Liu, WB, Zhou, DM: Global asymptotic stability to a generalized Cohen-Grossberg BAM neural networks of neutral type delays. Neural Netw. 25, 94-105 (2012)

13. Zhou, DM, Yu, SH, Zhang, ZQ: New LMl-based conditions for global exponential stability to a class of Cohen-Grossberg BAM networks with delays. Neurocomputing 121, 512-522 (2013)

14. Jian, JQ, Wang, BX: Global Lagrange stability for neutral-type Cohen-Grossberg BAM neural networks with mixed time-varying delays. Math. Comput. Simul. 116, 1-25 (2015)

15. Wang, DS, Huang, LH, Cai, ZW: On the periodic dynamics of a general Cohen-Grossberg BAM neural networks via differential inclusions. Neurocomputing 118, 203-214 (2013)

16. Yang, WG: Periodic solution for fuzzy Cohen-Grossberg BAM neural networks with both time-varying and distributed delays and variable coefficients. Neural Process. Lett. 40, 51-73 (2014)

17. Balasubramaniam, P, Syed Ali, M: Stability analysis of Takagi-Sugeno fuzzy Cohen-Grossberg BAM neural networks with discrete and distributed time-varying delays. Math. Comput. Model. 53, 151-160 (2011)

18. Li, XD, Fu, XL: Global asymptotic stability of stochastic Cohen-Grossberg-type BAM neural networks with mixed delays: an LMI approach. J. Comput. Appl. Math. 235, 3385-3394 (2011)

19. Li, YK, Chen, XR, Zhao, L: Stability and existence of a periodic solutions to delayed Cohen-Grossberg BAM neural networks with impulses on time scales. Neurocomputing 72, 1621-1630 (2009)

20. Li, XD: Exponential stability of Cohen-Grossberg-type bam neural networks with time-varying delays via impulsive control. Neurocomputing 73, 525-530 (2009)

21. Xiang, HJ, Cao, JD: Exponential stability of a periodic solution to Cohen-Grossberg-type BAM networks with time-varying delays. Neurocomputing 72, 1702-1711 (2009)

22. Mathiyalagan, K, Park, JH, Sakthivel, R: Synchronization for delayed memristive BAM neural networks using impulsive control with random nonlinearities. Appl. Math. Comput. 259, 967-979 (2015)

23. Mathiyalagan, K, Sakthivel, R, Anthoni, SM: Exponential stability result for discrete-time stochastic fuzzy uncertain neural networks. Phys. Lett. A 376, 901-912 (2012)

24. Sakthivel, R, Su, H, Shi, $P$, Sakthivel, R: Exponential $H_{\infty}$ filtering for discrete-time switched neural networks with random delays. IEEE Trans. Cybern. 45, 676-687 (2015)

25. Sakthivel, R, Vadivel, P, Mathiyalagan, K, Arunkumar, A, Sivachitra, M: Design of state estimator for bidirectional associative memory neural networks with leakage delays. Inf. Sci. 296, 263-274 (2015)

26. Syed Ali, M: Stability analysis of Markovian jumping stochastic Cohen-Grossberg neural networks with discrete and distributed time varying delays. Chin. Phys. B 23, 060702 (2014)

27. Syed Ali, M, Balasubramaniam, P, Zhu, QX: Stability of stochastic fuzzy BAM neural networks with discrete and distributed time-varying delays. Int. J. Mach. Learn. Cybern. (2015, in press). doi:10.1007/s13042-014-0320-7

28. Xu, CJ, Li, PL: Exponential stability for fuzzy BAM cellular neural networks with distributed leakage delays and impulses. Adv. Differ. Equ. 2016, 276 (2016)

29. Xu, CJ, Zhang, QM, Wu, YS: Existence and exponential stability of periodic solution to fuzzy cellular neural networks with distributed delays. Int. J. Fuzzy Syst. 18, 41-51 (2016)

30. Xu, CJ, Zhang, QM: Existence and stability of pseudo almost periodic solutions for shunting inhibitory cellular neura networks with neutral type delays and time-varying leakage delays. Netw. Comput. Neural Syst. 25, 168-192 (2014)

31. Li, KL, Zeng, HL: Stability in impulsive Cohen-Grossberg-type BAM neural networks with time-varying delays: a general analysis. Math. Comput. Simul. 80, 2329-2349 (2010)

32. Arbib, M: Branins, Machines, and Mathematics. Springer, New York (1987)

33. Zhou, L, Li, W, Chen, Y: Suppressing the periodic impulse in partial discharge detection based on chaotic control. Autom. Electr. Power Syst. 28, 90-94 (2004)

34. Nagesh, V: Automatic detection and elimination of periodic pulse shaped interference in partial discharge measurements. IEE Proc. Sci. Meas. Technol. 141, 335-339 (1994)

35. Bai, CZ: Stability analysis of Cohen-Grossberg BAM neural networks with delays and impulses. Chaos Solitons Fractals 35, 263-267 (2008)

36. Xia, YH: Impulsive effect on the delayed Cohen-Grossberg-type bam neural networks. Neurocomputing 73 , 2754-2764 (2010)

37. Yang, T, Yang, LB: The global stability of fuzzy cellular neural networks. IEEE Trans. Circuits Syst. 43, 880-883 (1996) 
38. Balasubramaniam, P, Syed Ali, M, Arik, S: Global asymptotic stability of stochastic fuzzy cellular neural networks with multiple time-varying delays. Expert Syst. Appl. 37, 7737-7744 (2010)

39. Li, YK, Wang, C: Existence and global exponential stability of equilibrium for discrete-time fuzzy BAM neural networks with variable delays and impulses. Fuzzy Sets Syst. 217, 62-79 (2013)

40. Li, XD, Rakkiyappan, R: Stability results for Takagi-Sugeno fuzzy uncertain BAM neural networks with time delays in the leakage term. Neural Comput. Appl. 22, 203-219 (2013)

41. Balasubramaniam, P, Vembarasan, V: Robust stability of uncertain fuzzy BAM neural networks of neutral-type with Markovian jumping parameters and impulses. Comput. Math. Appl. 62, 1838-1861 (2011)

42. Li, YT, Wang, JY: An analysis on the global exponential stability and the existence of a periodic solutions for non-autonomous hybrid BAM neural networks with distributed delays and impulses. Comput. Math. Appl. 56, 2256-2267 (2008)

43. Xu, DY, Yang, ZC: Impulsive delay differential inequality and stability of neural networks. J. Math. Anal. Appl. 305 107-120 (2005)

44. Zhou, QH, Wan, L: Impulsive effects on stability of Cohen-Grossberg-type bidirectional associative memory neural networks with delays. Nonlinear Anal., Real World Appl. 10, 2531-2540 (2009)

\section{Submit your manuscript to a SpringerOpen ${ }^{\circ}$ journal and benefit from:}

- Convenient online submission

- Rigorous peer review

- Immediate publication on acceptance

- Open access: articles freely available online

- High visibility within the field

- Retaining the copyright to your article 